\title{
X-RAY MICRO-CT SUPPORTING THE SOUTH AFRICAN ADDITIVE MANUFACTURING COMMUNITY
}

\author{
A. du Plessis ${ }^{1 *}$, S.G. le Roux ${ }^{2}$
}

\begin{abstract}
This paper presents the latest developments in microCT, both globally and locally, for supporting the additive manufacturing industry. There are a number of recently developed capabilities which are especially relevant to the non-destructive quality inspection of additive manufactured parts; and also for advanced process optimization. These new capabilities are all locally available but not yet utilized to their full potential, most likely due to a lack of knowledge of these capabilities. The aim of this paper is therefore to fill this gap and provide an overview of these latest capabilities, showcasing numerous local examples.
\end{abstract}

Keywords: Additive manufacturing; powder bed fusion; X-ray tomography; microCT; non-destructive testing; process optimization; porosity; pore hotspots; image-based simulations; 3D image analysis

1* Department of Physics, University of Stellenbosch, South Africa (Corresponding author: anton2@sun.ac.za )
2 CT Scanner Facility, University of Stellenbosch,

Presented at the 19th Annual International RAPDASA conference, held from 7 to 9 November 2018 in Johannesburg, South Africa. 


\section{INTRODUCTION}

Micro computed tomography (microCT) is now widely accepted as a powerful non-destructive testing method especially valuable for additively manufactured (AM) parts. As a non-destructive analysis method it has numerous industrial applications as reviewed in [1], where AM is also mentioned. The method had evolved over the last decade from a qualitative imaging technology to a quantitative materials science tool, with particular applications in understanding internal material structures [2]. It is widely known for its ability to visualize internal features, and is most widely known and used for porosity or flaw detection. This is particularly relevant to additive manufactured parts, as porosity and even major flaws might be present especially when process parameters are not optimized. The non-destructive inspection of complex AM parts is therefore crucial and is required for the qualification of such parts and the processes that produce them for particular applications [3]. The qualification and standardization needs for AM has been reviewed recently and microCT is a crucial aspect $[4,5]$. Besides the use of microCT for defect detection, many other microCT capabilities are still underutilized and some are still being developed as discussed in a recent review paper on the use of microCT in AM [6]. These new microCT capabilities have evolved due to recent improvements in hardware and software, especially with customized image analysis procedures, coupled with improvements in computing resources. These new capabilities include measurements of surface roughness, localized mean porosity calculations (to quantify porosity clustering), structural mechanics simulations to identify regions of high stress in real parts, and other image-based simulations based on data of the real part, amongst others. These latest advances were all mentioned in [6], but are discussed here in the context of their usage in local projects. Technical details are not included but details about the X-ray microCT facility and optimization of parameters may be found in $[7,8]$.

\section{POROSITY AND FLAW DETECTION}

The Stellenbosch microCT facility, which is described in more detail in [7] was first officially launched in April 2012, with interested AM users from CUT attending this event and providing test samples. In these first analyses of an AM Ti6Al4V part, porosity and layered defects were observed in the complex shaped part. This sample was known to have been produced under non-ideal conditions but it was not known if there were flaws present inside the part. This interesting layered defect was later published after additional HIPping of the same part, to see if these defects could be closed by the HIP process [9]. This type of defect is caused by stop-start cycles and are more recently referred to as stop-start flaws. In the same year (2012), another type of defect was observed: near-spherical pores arranged in 3D in a regularly-spaced checkerboard pattern. This was later reported in a paper [10] without details of its origin, as an example of directionality of porosity in AM parts. As shown in Figure 1 in a cross-sectional view, the pores are arranged in a checkerboard pattern in this case with a $0.2 \mathrm{~mm}$ spacing. This spacing corresponds roughly to the track width and might be the result of lack of fusion occurring between tracks in the same layer (between individual parallel tracks), but the subsequent 90 degree rotated layer of tracks does the same, effectively remelting the areas with porosity in the previous layer also, to leave only the intersection areas with pores. This requires further investigation and might be complicated by scan strategy but similar results were reported for different scan strategies also using microCT analysis in [11]. It was recently confirmed that there were scan errors in this system since 2012. Clearly the ability for microCT to detect and visualize the 3D distribution of the porosity might in future assist to more easily and quickly identify such manufacturing errors, and thereby ensure high density parts with good mechanical performance. This use of non-destructive testing has also been mentioned as part of process monitoring, for correlating in-situ detection with post-process analysis and thereby build a better understanding of the process [12]. More recently the production of AM parts with induced pores and cavities were used to test acoustic detection methods and used microCT to correlate the detection capability of the acoustic methods, as a "ground truthing" [13].

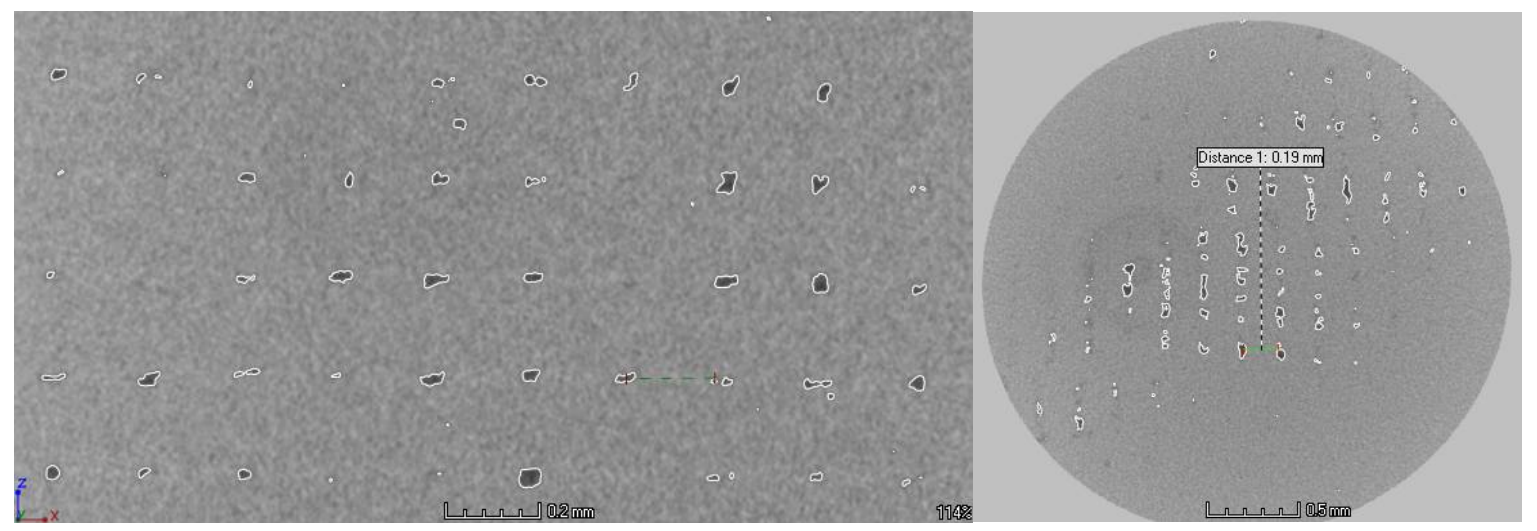

Figure 1: Two orthogonal thin slice views of the same sample showing regular pore spacing of $0.2 \mathrm{~mm}$ : (a) side view, (b) top view. 
These early examples showed that different forms of porosity or flaws may be present in AM parts and microCT may be the only way to fully visualize them and therefore identify the root cause of the problem, which is very useful for process parameter optimization and validation. Due to the complexity and large numbers of variables in the production process, problems may also emerge even when process parameters are optimized. Therefore the final built part should also be inspected, which can be done non-destructively. This may be critical for high performance parts and for medical implants. Over the last few years, medical implants produced by CUT have been tested often prior to implantation into the patient. The microporosity is always minimal and usually $<0.1 \%$ with mean pore sizes of $30-50 \mu \mathrm{m}$ as reported in [14]. However, there have been isolated cases where microCT identified critical flaws and the part has had to be built again. While a full implant cannot be analyzed at the highest possible resolution by microCT, it can identify major flaws such as stop-start flaws, and in case of such detection, the area of interest can be scanned at higher resolution for a more clear view of the extent of the defect (and possibly a more quantitative assessment). For this reason microCT forms part of the ISO accredited workflow for the production of additively manufactured implants at CUT.

The ability to detect porosity and internal voids in AM parts was investigated in detail with test parts produced and is reported in [15]. Furthermore, the shape of porosity in $A M$ parts was analyzed in tensile test parts at different stages of loading, indicating pore coalescence prior to tensile fracture [16].

\section{SURFACE SCANNING COMBINED WITH MICRO-CT}

This example showcases a new capability which is surface texture scanning, combined with microCT scanning. By scanning the same sample with a handheld scanner (Artec Eva or Spider) and by microCT allows the ability to produce realistic 3D textures of the surface while also allowing internal analysis of the microCT data. This is demonstrated on a bracket built in Ti6Al4V, shown in Figure 2. The main advantage of this new combination of data sets for additive manufacturing, is the cross-referencing capabilities - when a part is too large or dense, microCT data is noisy and might lose surface information in places - at these locations the surface scan could be used. On the other hand microCT is often more accurate and provides additional internal information and information from hard-to-reach locations.
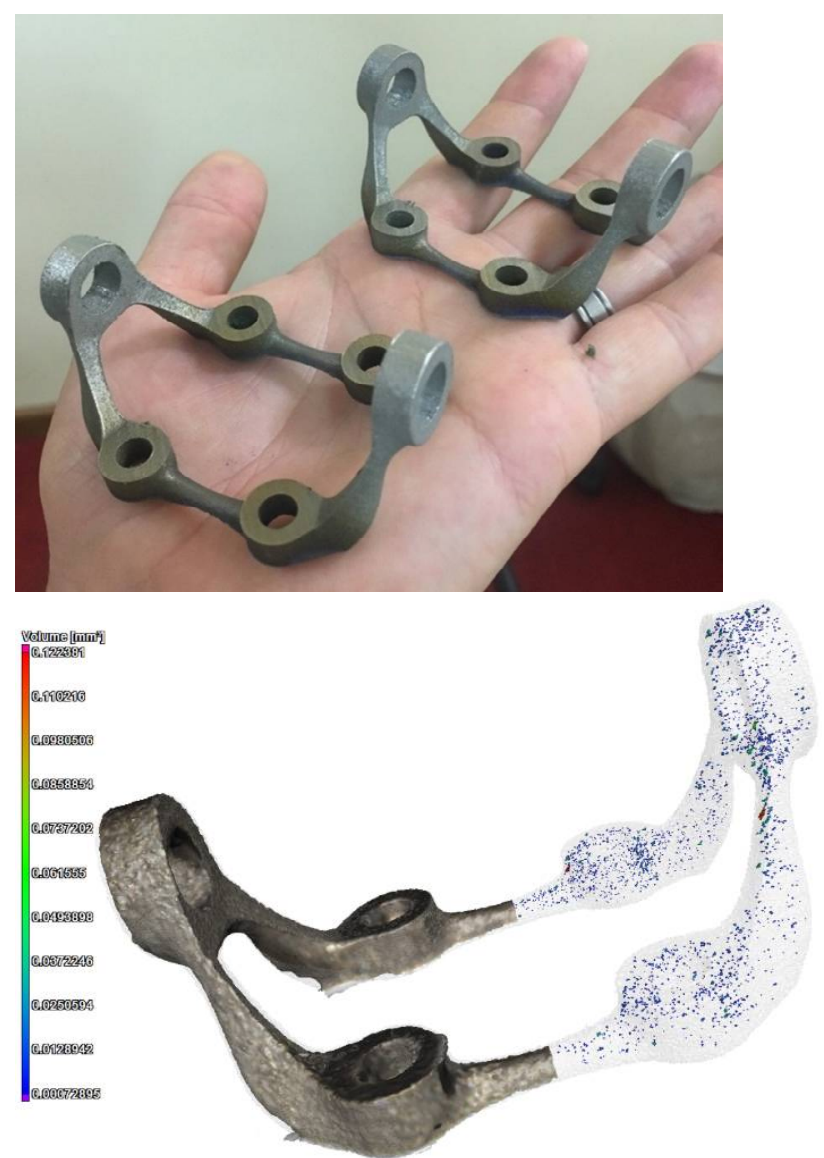

Figure 2: Surface scan combined with microCT - surface texture combined with microCT data is a new data merging capability. The bracket in the image to the right contains evenly distributed porosity at a level of $0.3 \%$. 


\section{POROSITY HOTSPOT ANALYSIS}

In the above example in Figure 2, evenly distributed porosity is seen in the bracket, at an average level of 0.3 $\%$. Even when the average porosity is much lower, the size and location of these pores are important, as they may be in a line, in a layer or clustered. Clustered pores may be analyzed by a new local porosity hotspot analysis which is demonstrated for AM parts for the first time here. The density of this part is excellent, and it contains minimal porosity at an average value of less than $0.01 \%$, but as shown in Figure 3 , the porosity is clustered right below the top surface, especially in flat areas - this indicates the origin of this type of porosity might be due to processing conditions in the finishing step, which are optimized for surface finish rather than microporosity.
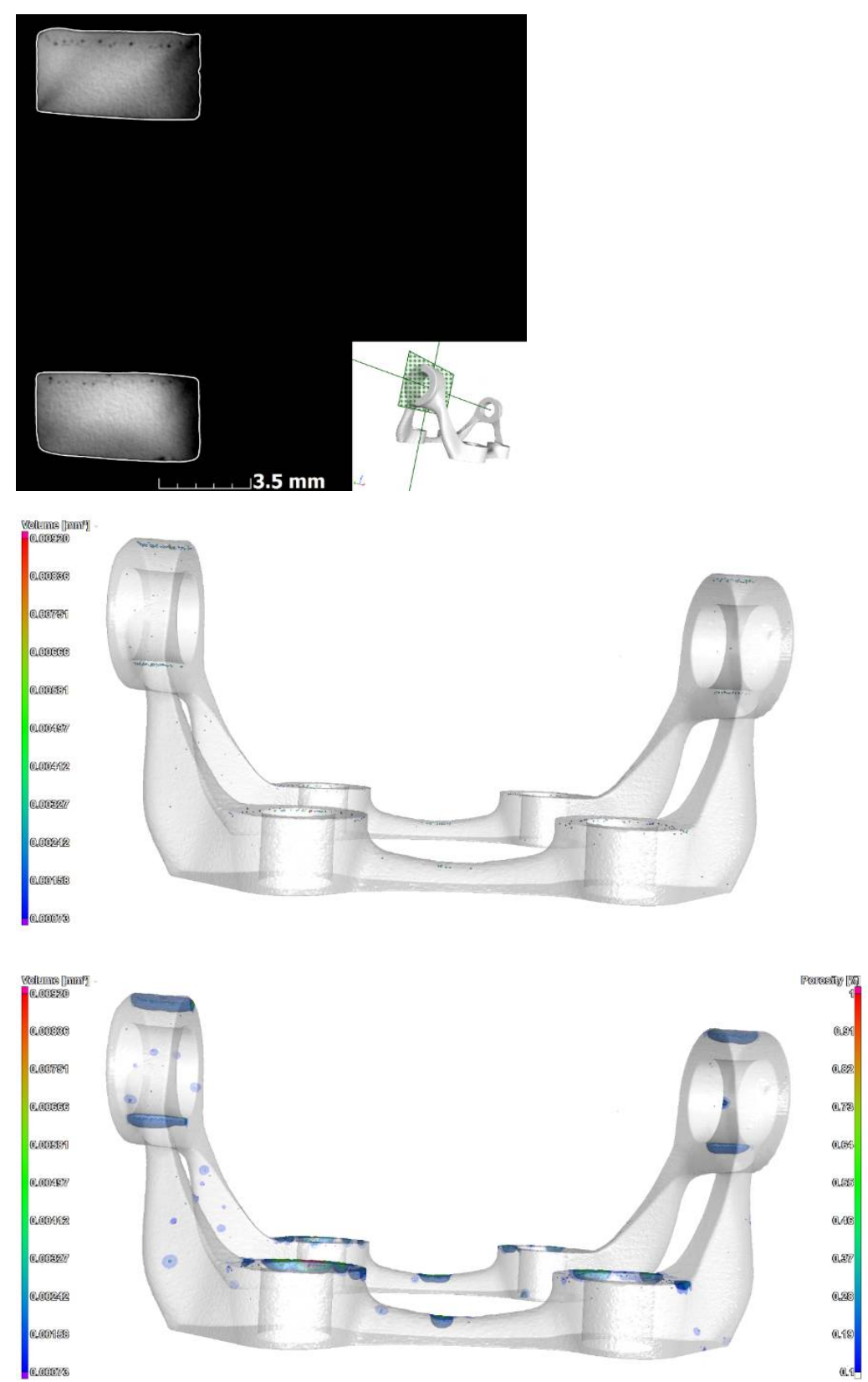

Figure 3: Localized porosity $0.5 \mathrm{~mm}$ under the top surface indicated by microCT slice image, 3D image and new hotspot analysis views, whereby localized high porosity is highlighted

\section{DENSITY}

While porosity analysis is popular, often samples are subjected to Archimedes tests for density. The Archimedes test is well known and very accurate for ideal samples - however there may be open porosity, inclusions, incorrect alloy content, or bubbles attaching to the rough surface of as-built parts, causing erroneous measurements. By using microCT data and sub-voxel precise surface determination, it is possible to calculate volume and hence density directly from microCT scans, without the need for detection of porosity 
in the images. This requires very high scan quality and advanced software tools to allow sub-voxel precision, something which is not always available and was not available at the Stellenbosch CT facility until 2014. Prior to this, various analyses were possible, but precision was limited to basic edge determination tools as shown in a Rapdasa proceedings paper in [17]. The advanced surface determination is demonstrated on a $10 \mathrm{~mm}$ Ti6Al4V cube in Figure 4, showing the 3D interpolation of grey values to determine the exact surface location - with high scan quality this can be as accurate as $1 / 10^{\text {th }}$ the voxel size. This allows accurate measurement of volumes, which allows effectively a direct microCT density measurement method.

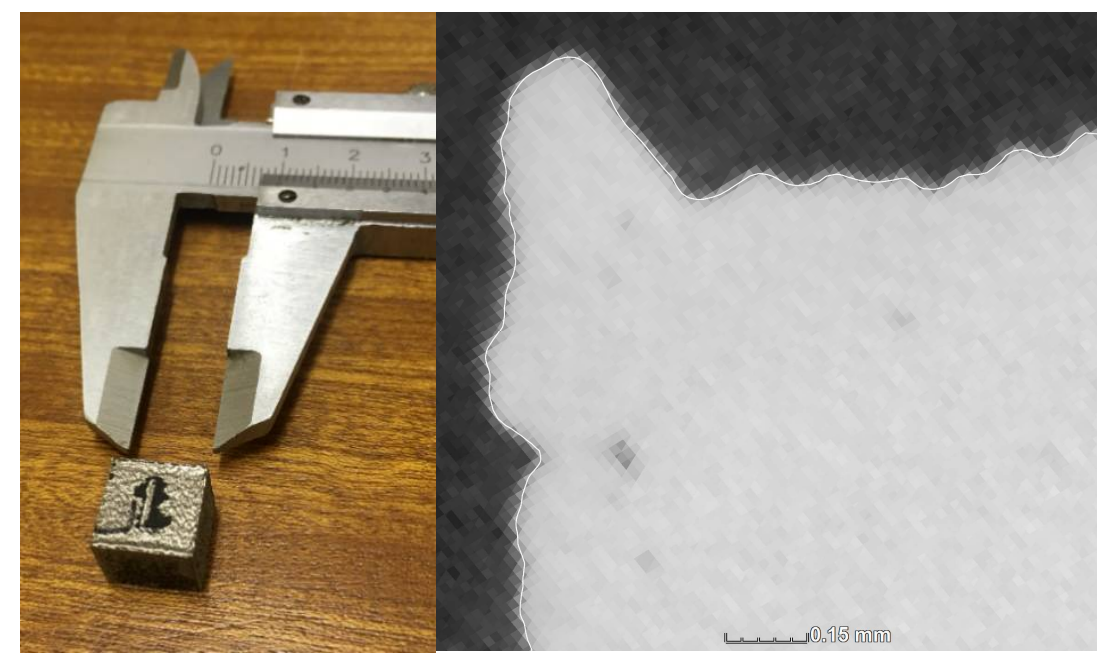

Figure 4: A $10 \mathrm{~mm}$ coupon sample, shown in a photo (left) and the corner of this sample in microCT slice image at $15 \mu \mathrm{m}$ voxel size including sub-voxel precise surface in white line.

\section{SURFACE ROUGHNESS}

Another important development is the capability to use a precise surface as shown above, and measure the surface roughness of as-built parts. This only works for small samples, for example 10-15 mm in size or diameter. When this is the case, the voxel size is approximately $15 \mu \mathrm{m}$, in which case it can be considered useful for typical "as-built" additive parts which have surfaces rougher than this value. Besides the advantage of area mapping for improved statistics (compared to traditional line-mapping for roughness), and the possibility to detect undercuts and hidden irregularities, even non-flat geometrical surfaces such as cylinders can be assessed as shown in Figure 5. This part was produced on an Optomec LENS system at CSIR.

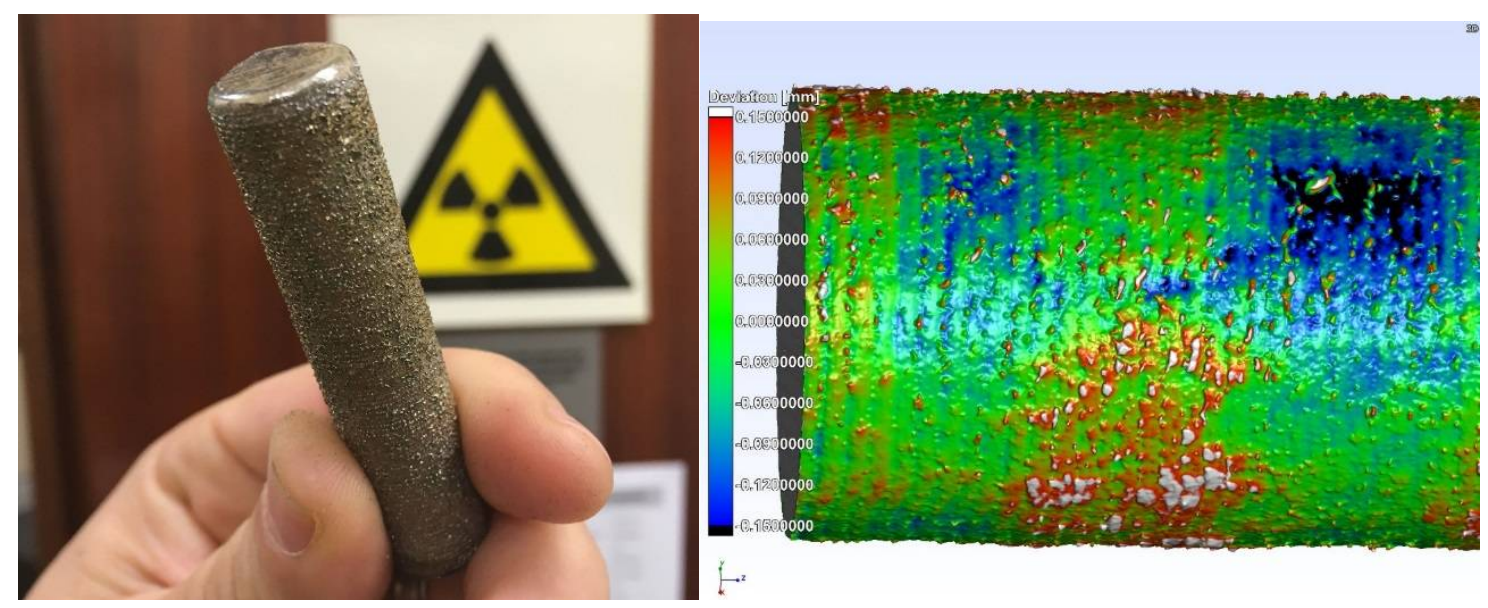

Figure 5: Surface roughness measurement, also on curved surfaces.

\section{DIMENSIONAL MEASUREMENT AND CAD VARIANCE}

The production of complex geometries bring with it challenges, such as residual stress which causes warping and possible cracks, and unexpected porosity or internal build defects. The bracket shown in Figure 6 is 
compared with its CAD design showing warping of up to $0.5 \mathrm{~mm}$ on the top parts (inwards). The STL design data is visualized as a wireframe in yellow in this case. It is also not so widely known that the microCT data can be easily exported in the form of a surface STL file. This simple data type and overlap of data is useful to assess the location of holes or important features in a built part, compared to its design. One important application of microCT in the near future might be analysis of the part geometry after HIP processing. HIPping is often used to close porosity, even very large pores can be closed effectively as shown in [18]. However, as shown in this study, near-surface pores might not be effectively closed, and these pores might be the critical ones for good fatigue life of the parts, as cracks may form between the surface and near-surface pores under cyclic loading. Furthermore, HIPping may force open near-surface pores, or cause other forms of macroscale deformations as shown in [19]. Such deformations may be analysed effectively by CAD variance analysis, and the additional benefit of using microCT is the ability to visualize the internal details (eg. is there a large pore sub-surface at the location of a surface indentation after HIP?).

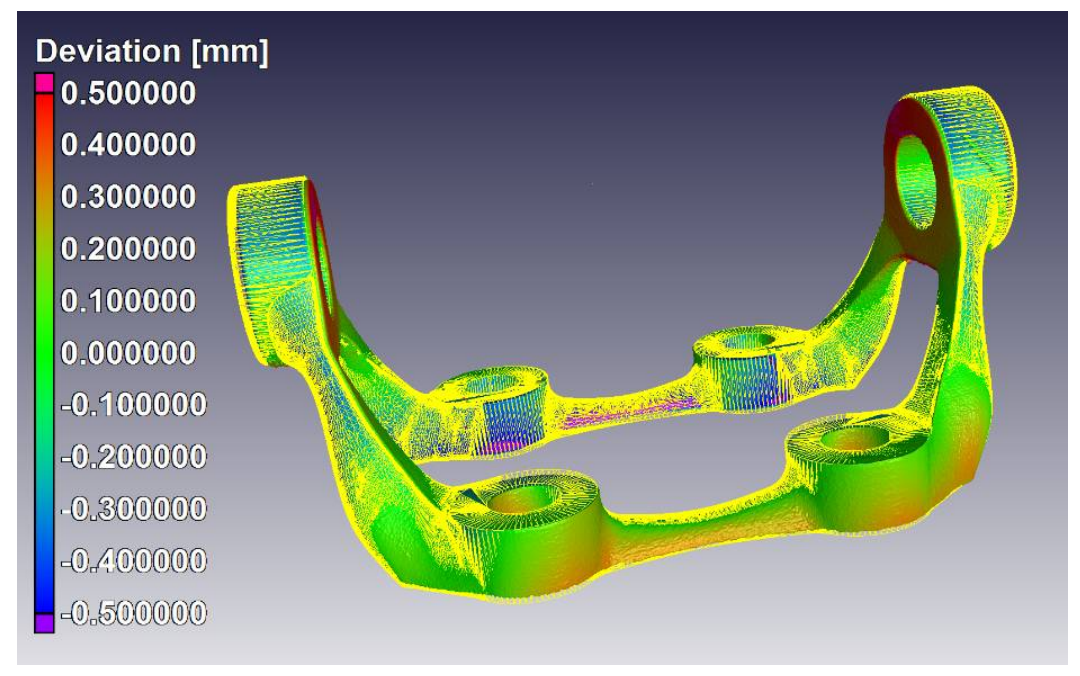

Figure 6: MicroCT analysis of complex parts for geometrical accuracy - using a CAD variance analysis. The yellow wireframe shows the CAD design.

\section{STRUCTURAL MECHANICS SIMULATIONS ON REAL PARTS}

Finite element modelling (FEM) is often used to assess a new design, but it has also recently become possible to directly make such simulations on microCT data. As shown in Figure 7, a jawbone implant was produced by $\mathrm{AM}$, containing microporosity and its exterior as-built geometry was significantly different than designed. When a simulation is applied, the stress distribution can be analysed and at the locations of high stress, the interior can be assessed for porosity and wall thickness, to assess the risk in using the part. This method therefore uses the actual geometry of the part and its internal pores, which provides a more accurate estimate of the stress distributions and displacements across the part, assuming linear elastic material properties. This method has not yet been used widely but was recently used in a study of biomimetic design of lattice structures [20] and its use is well documented in supplementary material in [21]. The method was also used to analyse the effect of large casting porosity on the stress distribution and resulting mechanical properties of cast Ti6Al4V in [22]. While this latter work is not focused on additive manufacturing, the effect of pores on the mechanical properties is very relevant and this work showed that even large pores (4 mm diameter) do not strongly affect the static tensile strength of cylindrical tensile bars $8 \mathrm{~mm}$ in diameter, when the pores are deep inside the material (not on surface). This is not a general statement but indicates the added value brought about by direct simulation including pores, especially for complex geometries and irregular pores. The stress concentrations found by these simulations might be indicative of fatigue life also as demonstrated with the same method applied to additive manufactured brackets in [23]. 


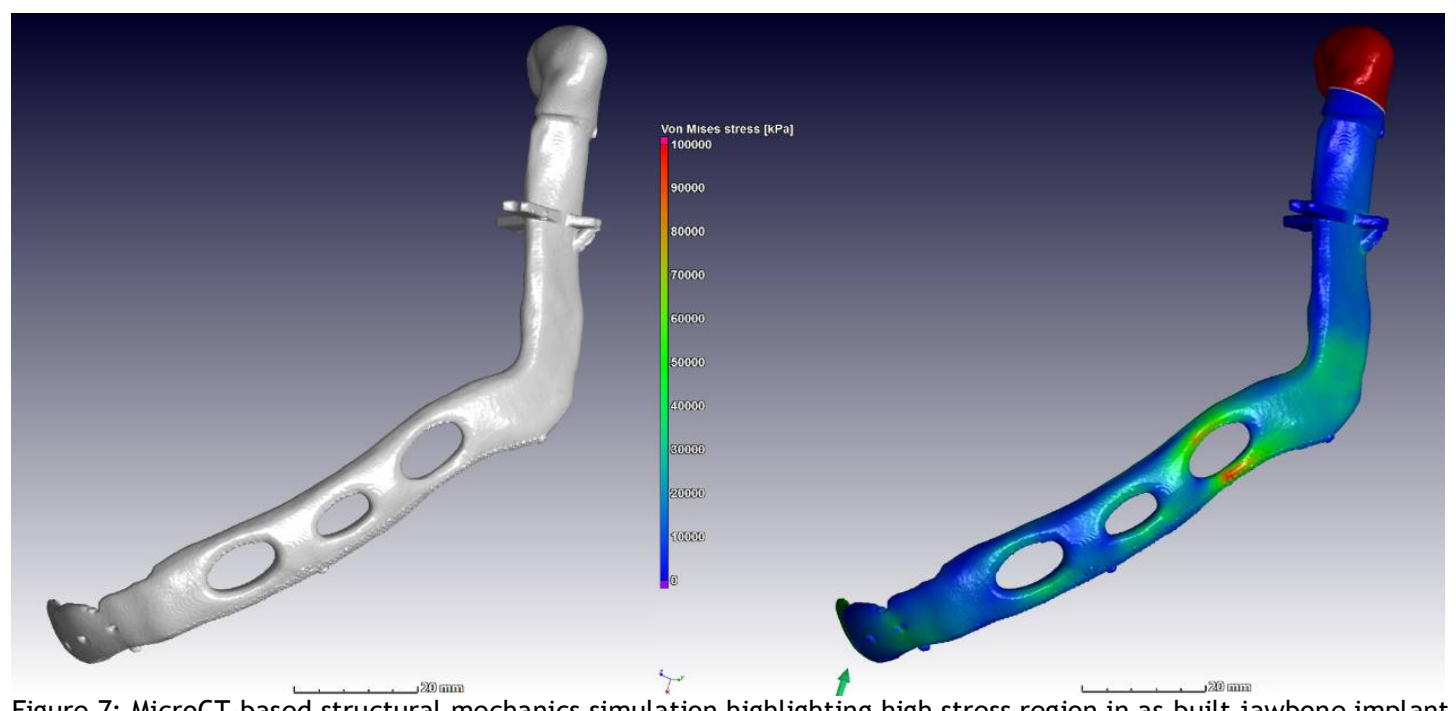

Figure 7: MicroCT-based structural mechanics simulation highlighting high stress region in as-built jawbone implant

\section{OTHER IMAGE BASED SIMULATIONS}

Other image-based simulations are also relevant to additive manufacturing, especially the simulation of permeability. This was recently used in a study comparing various lattice structure designs meant for additive manufacturing [24]. An example is shown in Figure 8 of a minimal surface unit cell design, with permeability simulation showing velocity flow lines. It is also possible to make such simulations on real produced sample data, thereby incorporating rough surfaces, warping or unconsolidated powder stuck inside the lattice.
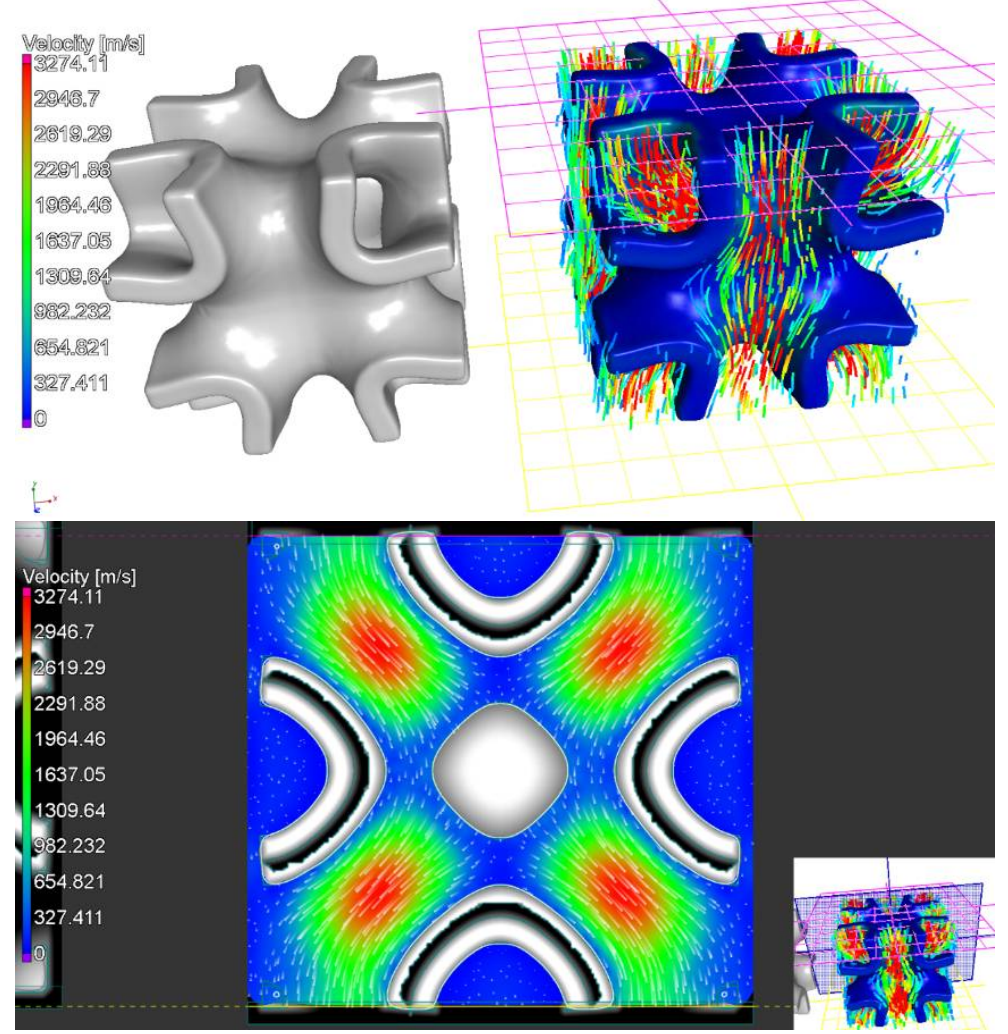

Figure 8: Permeability simulations directly on voxel data.

\section{ADVANCED MORPHOLOGICAL ANALYSIS}

Some advanced methods of analysis are possible for application on structures such as lattices produced by additive manufacturing - in the image in Figure 9, individual struts are selected and thickness analysis applied using a method measuring the largest sphere that fits the strut. In this way it can be easily visualized that the 
vertical strut is thinner than the horizontal strut, and the values can be quantitatively measured for comparing different samples, for example.

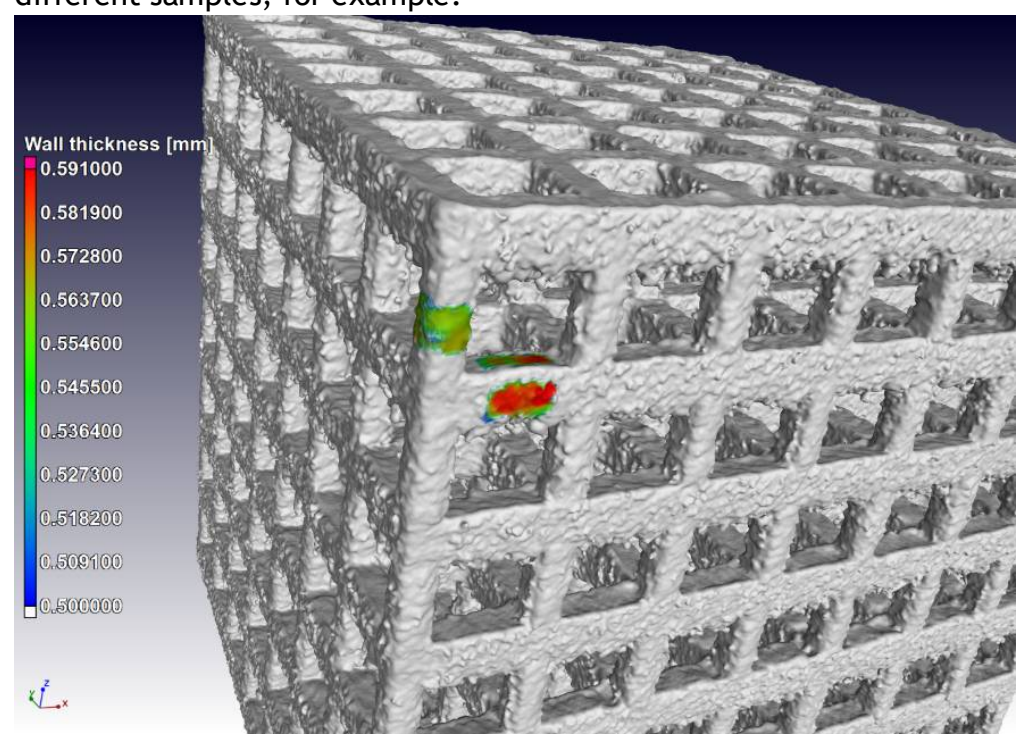

Figure 9: Thickness analysis of horizontal and vertical struts in a cubic lattice structure

\section{PLASTICS}

Since 3D printing of plastics is mainly used for prototyping and not for critical parts, microCT is not widely used for this type of material. However, there have been some local projects where ABS and PLA filaments were analyzed by microCT showing the presence of dense particles which could block the nozzle [25] - present in some filament types. In another study the quality of 3D printed models were compared by microCT for two different filament types on two different 3D printers [26]. Since plastics are used for prototypes, they can be used to better visualize 3D models especially from microCT scans of interesting objects such as museum specimens as shown for a mummy falcon $[27,28]$ and more recently to visualize a hairy stalagmite - a complex biological root system in a stalagmite, of which a part of the model was 3D printed to better understand the complex interconnections [29].

\section{AUTOMATION AND STANDARDIZATION}

One of the major drawbacks of microCT is its cost and complexity as each part needs custom analysis workflows. In a recent development, a series of methods were developed for simplified analysis workflows using standard sample sizes, scan settings and image analysis procedures. These may contribute to the higher usage and lower cost of the technique, and its more productive use in additive manufacturing. The methods involve the production of a $10 \mathrm{~mm}$ cube, which is used for detailed microporosity analysis - this can be used for process optimization and validation [30]. The same cube than then also be analysed for its bulk density using microCT volume and scale mass [31]. The same data can be used to assess surface roughness as demonstrated in [32]. A $15 \mathrm{~mm}$ rod can be built during the production of a complex part and this witness specimen can be scanned with fixed parameters irrespective of the complex part size - this also contributes to standardization in the analysis process and allows detection of potential layered flaws [33]. A standard method for analysis of powders was also developed [34]. The automation of the analysis workflow will greatly contribute to higher throughput, but this is still under development. A first round robin test were parts were produced at various facilities and tested using these standard methods was completed and can be found in [35]. A second round robin test is currently underway, where 3 selected parts from this first test, is analysed at various microCT facilities worldwide using the standardized workflows.

\section{CONCLUSIONS}

This paper has shown the many ways in which microCT can support the AM community, with a variety of new methods that have recently been developed. South African examples are provided of each of these methods, with some of them being the first such cases reported in scientific literature. Furthermore, interesting use cases of microCT in $A M$ over the last few years in South Africa were highlighted. Not all projects were mentioned as some were not published and many were meant only as preliminary investigations. The development of standardization techniques was discussed and work in progress in this domain is set to improve the simplicity and ease of use of the technique. MicroCT will continue to support the quality inspection and improvement of AM part integrity. 


\section{REFERENCES}

[1] L. De Chiffre, S. Carmignato, J.P. Kruth, R. Schmitt, A. Weckenmann, CIRP Annals Manufacturing Technology Industrial applications of computed tomography, Cirp Ann. - Manuf. Technol. - 2014, Vol. 63, Issue 2, Pp. 655-677. (2014) 1-25.

[2] E. Maire, P.J. Withers, Quantitative X-ray tomography, Int. Mater. Rev. 59 (2014) 1-43. doi:10.1179/1743280413Y.0000000023.

[3] I. Yadroitsev, P. Krakhmalev, I. Yadroitsava, A. Du Plessis, Qualification of Ti6Al4V ELI Alloy Produced by Laser Powder Bed Fusion for Biomedical Applications, JOM. 70 (2018) 372-377. doi:10.1007/s11837017-2655-5.

[4] M. Seifi, A. Salem, J. Beuth, O. Harrysson, J.J. Lewandowski, Overview of Materials Qualification Needs for Metal Additive Manufacturing, JOM. 68 (2016) 747-764. doi:10.1007/s11837-015-1810-0.

[5] M. Seifi, M. Gorelik, J. Waller, N. Hrabe, N. Shamsaei, S. Daniewicz, J.J. Lewandowski, Progress Towards Metal Additive Manufacturing Standardization to Support Qualification and Certification, JOM. 69 (2017) 439-455. doi:10.1007/s11837-017-2265-2.

[6] A. du Plessis, I. Yadroitsev, I. Yadroitsava, S.G. Le Roux, X-Ray Microcomputed Tomography in Additive Manufacturing: A Review of the Current Technology and Applications, 3D Print. Addit. Manuf. 0 (2018). doi:10.1089/3dp.2018.0060.

[7] A. du Plessis, S.G. le Roux, A. Guelpa, The CT Scanner Facility at Stellenbosch University: An open access X-ray computed tomography laboratory, Nucl. Instruments Methods Phys. Res. Sect. B Beam Interact. with Mater. Atoms. 384 (2016) 42-49. doi:10.1016/j.nimb.2016.08.005.

[8] A. du Plessis, C. Broeckhoven, A. Guelpa, S.G. le Roux, Laboratory x-ray micro-computed tomography: A user guideline for biological samples, Gigascience. 6 (2017) 42-49. doi:10.1093/gigascience/gix027.

[9] A. du Plessis, S.G. le Roux, J. Els, G. Booysen, D.C. Blaine, Application of microCT to the nondestructive testing of an additive manufactured titanium component, Case Stud. Nondestruct. Test. Eval. 4 (2015) 1-7. doi:10.1016/j.csndt.2015.09.001.

[10] A. du Plessis, S.G. le Roux, G. Booysen, J. Els, Directionality of Cavities and Porosity Formation in Powder-Bed Laser Additive Manufacturing of Metal Components Investigated Using X-Ray Tomography, 3D Print. Addit. Manuf. (2016). doi:10.1089/3dp.2015.0034.

[11] D. Hagedorn-Hansen, M. Bezuidenhout, D. Dimitrov, The effects of selective laser melting scan strategies on deviation of hybrid parts, (2017). http://scholar.sun.ac.za/handle/10019.1/102998 (accessed June 21, 2018).

[12] M. Schmidt, M. Merklein, D. Bourell, D. Dimitrov, T. Hausotte, K. Wegener, L. Overmeyer, F. Vollertsen, G.N. Levy, Laser based additive manufacturing in industry and academia, CIRP Ann. 66 (2017) 561-583. doi:10.1016/J.CIRP.2017.05.011.

[13] D. Kouprianoff, N. Luwes, E. Newby, I. Yadroitsava, I. Yadroitsev, On-line monitoring of laser powder bed fusion by acoustic emission: Acoustic emission for inspection of single tracks under different powder layer thickness, in: 2017 Pattern Recognit. Assoc. South Africa Robot. Mechatronics, IEEE, 2017: pp. 203-207. doi:10.1109/RoboMech.2017.8261148.

[14] A. Du Plessis, S.G. Le Roux, G. Booysen, J. Els, Quality Control of a Laser Additive Manufactured Medical Implant by X-Ray Tomography, 3D Print. Addit. Manuf. 3 (2016) 175-182. doi:10.1089/3dp.2016.0012.

[15] D. Kouprianoff, A. Du Plessis, I. Yadroitsava, I. Yadroitsev, NON-DESTRUCTIVE TESTING OF THE PARTS MANUFACTURED BY DIRECT METAL LASER SINTERING, (n.d.).

[16] P. Krakhmalev, G. Fredriksson, I. Yadroitsava, N. Kazantseva, A. Du Plessis, I. Yadroitsev, Deformation behavior and microstructure of Ti6Al4V manufactured by SLM, in: Phys. Procedia, 2016. doi:10.1016/j.phpro.2016.08.080.

[17] A. du Plessis, T. Seifert, G. Booysen, J. Els, Microfocus X-ray computed tomography (CT) analysis of laser sintered parts, South African J. Ind. Eng. 25 (2014) 39-49.

[18] A. du Plessis, P. Rossouw, Investigation of Porosity Changes in Cast Ti6Al4V Rods After Hot Isostatic Pressing, J. Mater. Eng. Perform. 24 (2015) 3137-3141. doi:10.1007/s11665-015-1580-4.

[19] A. du Plessis, P. Rossouw, X-ray computed tomography of a titanium aerospace investment casting, Case Stud. Nondestruct. Test. Eval. 3 (2015) 21-26. doi:10.1016/j.csndt.2015.03.001.

[20] A. du Plessis, C. Broeckhoven, I. Yadroitsev, I. Yadroitsava, S.G. le Roux, Analyzing nature's protective design: The glyptodont body armor, J. Mech. Behav. Biomed. Mater. 82 (2018). doi:10.1016/j.jmbbm.2018.03.037.

[21] A. Du Plessis, C. Broeckhoven, S.G. Le Roux, Snake fangs: 3D morphological and mechanical analysis by microCT, simulation, and physical compression testing, Gigascience. 7 (2018) 1-8. doi:10.1093/gigascience/gix126.

[22] A. du Plessis, I. Yadroitsava, S.G. le Roux, I. Yadroitsev, J. Fieres, C. Reinhart, P. Rossouw, Prediction of mechanical performance of Ti6Al4V cast alloy based on microCT-based load simulation, J. Alloys Compd. 724 (2017). doi:10.1016/j.jallcom.2017.06.320. 
[23] J. Fieres, P. Schumann, C. Reinhart, Predicting failure in additively manufactured parts using X-ray computed tomography and simulation, Procedia Eng. 213 (2018) 69-78. doi:10.1016/J.PROENG.2018.02.008.

[24] A. du Plessis, I. Yadroitsava, I. Yadroitsev, S. le Roux, D. Blaine, Numerical comparison of lattice unit cell designs for medical implants by additive manufacturing, Virtual Phys. Prototyp. In press (2018).

[25] A. Du Plessis, S.G. Le Roux, F. Steyn, Quality Investigation of 3D Printer Filament Using Laboratory XRay Tomography, 3D Print. Addit. Manuf. 3 (2016) 262-267. doi:10.1089/3dp.2016.0011.

[26] A. du Plessis, S.G. le Roux, F. Steyn, X-Ray Computed Tomography of Consumer-Grade 3D-Printed Parts, 3D Print. Addit. Manuf. 2 (2015) 190-195. doi:10.1089/3dp.2015.0015.

[27] A. Du Plessis, R. Slabbert, L.C. Swanepoel, J. Els, G.J. Booysen, S. Ikram, I. Cornelius, Threedimensional model of an ancient Egyptian falcon mummy skeleton, Rapid Prototyp. J. 21 (2015) 368372. doi:10.1108/RPJ-09-2013-0089.

[28] S. Ikram, R. Slabbert, I. Cornelius, A. du Plessis, L.C. Swanepoel, H. Weber, Fatal force-feeding or Gluttonous Gagging? The death of Kestrel SACHM 2575, J. Archaeol. Sci. 63 (2015) 72-77. doi:10.1016/j.jas.2015.08.015.

[29] G. Du Preez, P. Forti, Hairy Stalagmites, a new biogenic root speleothem from Botswana, Int. J. .... (2015). $\quad$ http://search.proquest.com/openview/a7459222787f6a30916510d5d2490d43/1?pqorigsite $=$ gscholar (accessed April 11, 2016).

[30] A. du Plessis, Standard method for microCT-based additive manufacturing quality control 1: Porosity analysis, Protocols.lo. (2018). https://www.protocols.io/view/standard-method-for-microct-basedadditive-manufac-sc7eazn?abstract.

[31] A. du Plessis, Standard method for microCT-based additive manufacturing quality control 2: density measurement, Protocols.lo. (2018). https://www.protocols.io/view/standard-method-for-microctbased-additive-manufac-sbvean6.

[32] A. du Plessis, Standard method for microCT-based additive manufacturing quality control 3: surface roughness, Protocols.lo. (2018). https://www.protocols.io/view/standard-method-for-microct-basedadditive-manufac-sbweape.

[33] A. du Plessis, Standard method for microCT-based additive manufacturing quality control 5: witness specimen, Protocols.lo. (2018). https://www.protocols.io/view/standard-method-for-microct-basedadditive-manufac-sbzeap6.

[34] A. du Plessis, Standard method for microCT-based additive manufacturing quality control 4: powder characterization, Protocols.lo. (2018). https://www. protocols.io/view/standard-method-for-microctbased-additive-manufac-sbyeapw.

[35] A. du Plessis, S.G. le Roux, Standardized X-ray tomography testing of additively manufactured parts: a round robin test, Addit. Manuf. In press (2018). 